\title{
A. baumannii histone acetyl transferase Hpa2: Optimization of homology modeling, analysis of protein-protein interaction and virtual screening
}

\author{
Jyoti Singh Tomar* and Rama Krishna Peddinti* \\ Department of Chemistry, Indian Institute of Technology Roorkee \\ Roorkee-247 667, Uttarakhand, India
}

E-mail: rkpedfcy@,iitr.ac.in, ramakpeddinti@gmail.com, manutomar1@gmail.com

\section{ELECTRONIC SUPPLEMENTARY INFORMATION}

Figure S1. A) Multiple sequence alignment of Hpa2 sequence with its templates having sequence similarity up to 29\%, B) Multiple sequence alignment of Hpa2 sequence with 1XEB and 1QSM protein templates. (the area highlighted in red represents the residues conserved between all the aligned proteins, and that in yellow represents the residues similar in nature, purple box is showing the motif residues of GNAT

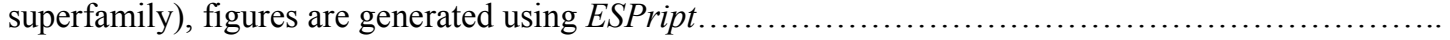

Table S1. Summary of structural validation programs and parameters values for Hpa2 monomer model generated by the Swiss-model/Modeller programs and optimized model using FoldX.......

Figure S2. Docked pose of acetyl-CoA (magenta) superimposed on the experimental pose (from yeast Hpa2 i.e., 1QSM Red) showing interactions with motif residues (Blue coloured) $Q \backslash R-X-X-G-X-G \backslash A$ of GNAT superfamily

Table S2. $\quad$ PDB files, residues involved in the formation of experimental dimer interface and rmsd values for redocked experimental dimers calculated using experimental structure as reference......

Figure S3. Experimental dimeric structures redocked using the data-driven molecular docking. The residues involved in the interface formation are labelled.

Table S3. ZINC_ID and binding affinities of the best ten compounds with monomer as well as dimer obtained through virtual screening (out of 760 which are obtained from ZINC database through substrate structure based search)

Figure S4. A) Experimental pose of acetyl-CoA (magenta: 1GHE) superimposed on the experimental pose (blue: 1QSM), showing differences in the conformation having optimal binding interactions. 1GHE protein is represented as cyan colour cartoon. B) Redocked pose of acetyl-CoA (magenta) superimposed on the experimental pose (blue: 1QSM) of yeast Hpa2.

Figure S5. A) Structures of best ten compounds showing good binding with monomer in virtual screening. B) Structures of best ten compounds showing good binding with dimer obtained in virtual screening. .....

Figure S6. Redocked pose of acetyl-CoA (magenta) superimposed on inhibitors docked pose (blue: ZINC08215434, green: ZINC85629887, cyan: ZINC65731330 and marine: ZINC85564657) at the model active site (green cartoon) to depict the similarity in binding interactions.... 
Figure S1 A) Multiple sequence alignment of Hpa2 sequence with its templates having sequence similarity up to $29 \%$, B) Multiple sequence alignment of Hpa2 sequence with 1XEB and 1QSM protein templates, (the area highlighted in red represents the residues conserved between all the aligned proteins, and that in yellow represents the residues similar between at least two of the aligned proteins, purple box is showing the motif residues of GNAT superfamily), figures are generated using ESPript.

A)

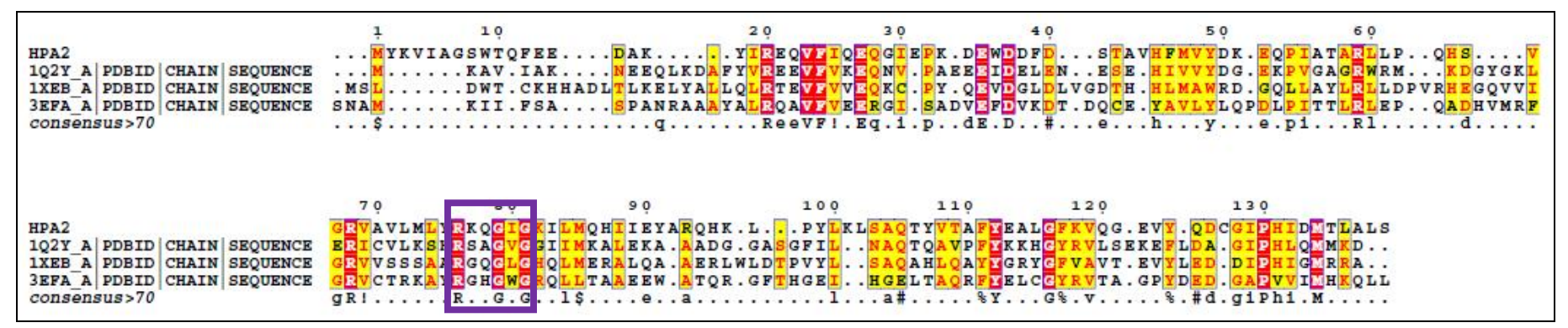

B)

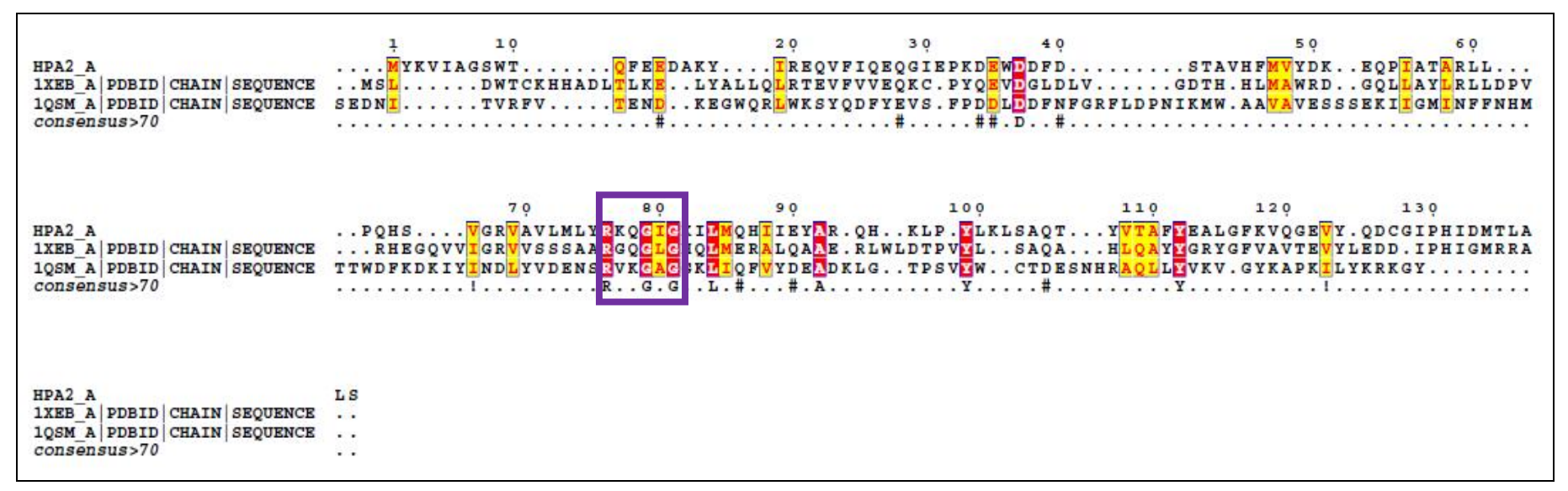


Table S1. Summary of structural validation programs and parameters values for Hpa 2 monomer model generated by the Swiss-model/Modeller programs and optimized model using FoldX.

\begin{tabular}{clccc}
\hline \multirow{2}{*}{ Server } & \multicolumn{1}{c}{ Parameters } & $\begin{array}{c}\text { Hpa2 Model } \\
\text { (Swiss-model) }\end{array}$ & $\begin{array}{c}\text { Hpa2 Model } \\
\text { (Modeller) }\end{array}$ & $\begin{array}{c}\text { Repaired } \\
\text { Hpa2 Model }\end{array}$ \\
\hline \multirow{2}{*}{ PROCHECK } & Residues in core, Allowed & $85.1 \%, 11.1 \%$ & $82.8 \%, 12.6 \%$ & $85.4 \%, 10.7 \%$ \\
& Generously allowed and & $2.5 \%$ & $3.4 \%$ & $2.5 \%$ \\
\cline { 2 - 2 } & Disallowed regions & $1.4 \%$ & $1.2 \%$ & $1.4 \%$ \\
\multirow{2}{*}{ Verify3D } & PROSA Z-score & -5.75 & -5.70 & -5.75 \\
Errat & 1D score > 0.2 & & & \\
& Overall quality factor & $75.01 \%$ & $66.13 \%$ & $88.24 \%$ \\
\hline
\end{tabular}

Figure S2. Docked pose of acetyl-CoA (magenta) superimposed on the experimental pose (from yeast Hpa2 i.e., 1QSM Red) showing interactions with motif residues (Blue coloured) $Q|R-X-X-G-X-G| A$ of GNAT superfamily.

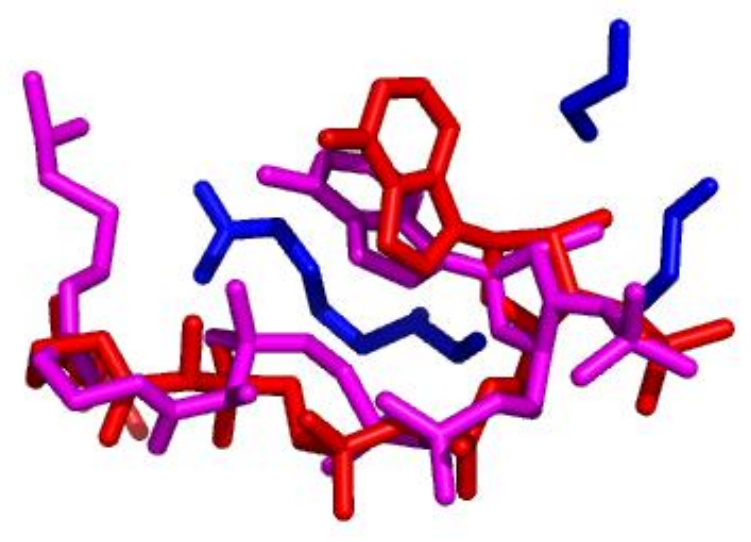


Table S2. PDB files, residues involved in the formation of experimental dimer interface and rmsd values for redocked experimental dimers calculated using experimental structure as reference.

\begin{tabular}{|c|c|c|}
\hline $\begin{array}{l}\text { PDB/ } \\
\text { MODEL }\end{array}$ & Interface residues & $\begin{array}{l}\text { RMSD } \\
\text { value }\end{array}$ \\
\hline 1XEB-AC & $\begin{array}{l}\text { A-Chain: H72, E73, G74, Q75, V77, Y114, V133, T134, Y137 and R148. } \\
\text { C-Chain: V70, E73, G74, Q75, V77, Y114, V133, T134, and R148. }\end{array}$ & 3.02 \\
\hline $1 \mathrm{~J} 4 \mathrm{~J}-\mathrm{AB}$ & $\begin{array}{l}\text { A-Chain: Q16, L17, Q121, D124, E125, E127, Q128, K182 and L184. } \\
\text { B-Chain: Q16, L17, R18, T21, E23, H27, Y28, A79 and D81. }\end{array}$ & 2.35 \\
\hline 1GHE-AB & $\begin{array}{l}\text { A-Chain: H30, G31, P100, S101, R103, R105 and V136. } \\
\text { B-Chain: H19, Q23, D42, M43, Q44 and Y47. }\end{array}$ & 3.23 \\
\hline 2DXQ-AB & $\begin{array}{l}\text { A-Chain: L16, Q27, D30, P31, L33, A38, V41, A44, M45, P49, L51, V71, P72, } \\
\text { N73, L74, T75, R76, A77, A78, R79, Y81, F83, F111, C115, Y116, V118, M119, } \\
\text { L121, T122, Y133, F138, V139, K142, T143, F145, Q146, I147, R148 and G149. } \\
\text { B-Chain: L16, Q27, D30, P31, L33, A38, V41,G48, P49, P72, N73, L74, R76, } \\
\text { A77, A78, R79, Y81, F83, N86, R104, Y116, K117, V118, M119, L120, L121, } \\
\text { T122, Q140, V139, N141, K142, T143, F145, Q146, I147, and R148. }\end{array}$ & 6.80 \\
\hline \multirow[t]{2}{*}{ 1QSM-AB } & $\begin{array}{l}\text { A-Chain:Y29, Y33, V35, F37, P38, D40, L41, F44, A45, R48, F49, I54, K55, } \\
\text { M56, N74, F76, M79, T80, T81, W82, D83, F84, K85, D86, K87, Y89, N91, } \\
\text { D92, Q110, Y113, D114, A116, D117, P122, S123, V124, Y125, W126, C127, } \\
\text { T128, D129, E130, S131, Q136, Y139, V140, K141, V142, Y144, K145, A146, } \\
\text { P147, K148, I149, L150, Y151, K152, R153, K154 and Y156. }\end{array}$ & 4.79 \\
\hline & $\begin{array}{l}\text { B-Chain: W26, Y29, Y33, V35, F37, P38, D40, L41, F44, N45, R48, F49, I54, } \\
\text { K55, M56, N74, F76, N77, M79, T80, T81, W82, D83, F84, K85, D86, K87, } \\
\text { Y89, N91, D92, Q110, Y113, D114, A116, D117, P122, S123, V124, Y125, } \\
\text { W126, C127, T128, D129, E130, Q136, Y139, V140, K141, V142, Y144, K145, } \\
\text { A146, P147, K148, I149, Y151, K152, R153, K154 and Y156. }\end{array}$ & \\
\hline Dimer-Hpa2 & $\begin{array}{l}\text { A-Chain: } \mathrm{Q} 63, \mathrm{H} 64, \mathrm{P} 98, \mathrm{Y} 99, \mathrm{G} 121, \mathrm{D} 122, \mathrm{~V} 123, \mathrm{Y} 124 \text { and D133. } \\
\text { B-Chain: } \mathrm{D} 38, \mathrm{~F} 39, \mathrm{~T} 42, \mathrm{~L} 61, \mathrm{P} 62, \mathrm{Q} 63, \mathrm{H} 64, \mathrm{~V} 123, \mathrm{Y} 124, \mathrm{Q} 125 \text { and D126. }\end{array}$ & 4.41 \\
\hline
\end{tabular}


Figure S3. Experimental dimeric structures redocked using the data-driven molecular docking. The residues involved in the interface formation are labelled.
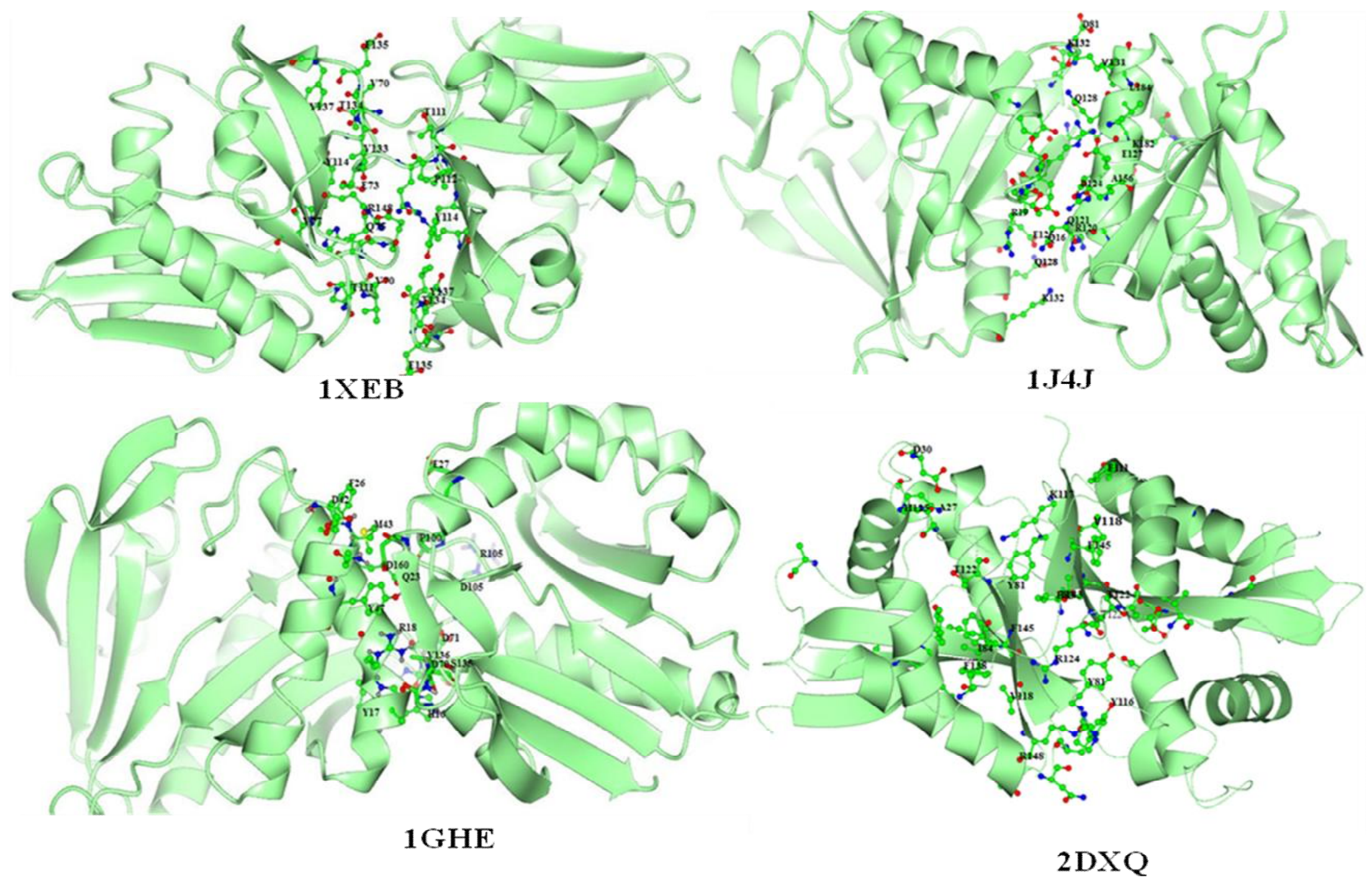
Table S3. ZINC_ID and binding affinities of the best ten compounds with monomer as well as dimer obtained through virtual screening (out of 760 which are obtained from ZINC database through substrate structure based search). The compounds in Red exhibit good binding affinity at both the binding sites.

\begin{tabular}{cccc}
\hline $\begin{array}{c}\text { Compounds binding at } \\
\text { interface of dimer }\end{array}$ & $\begin{array}{c}\text { Binding energy } \\
\text { (kcal/mol) }\end{array}$ & $\begin{array}{c}\text { Compounds binding at } \\
\text { active site of monomer }\end{array}$ & $\begin{array}{c}\text { Binding energy } \\
\text { (kcal/mol) }\end{array}$ \\
\hline ZINC65731330 & -12.10 & ZINC43411693 & -10.20 \\
ZINC08215434 & -12.10 & ZINC85629877 & -10.10 \\
ZINC85564657 & -12.00 & ZINC94303217 & -09.90 \\
ZINC85629877 & -11.90 & ZINC43411702 & -09.90 \\
ZINC85564663 & -11.80 & ZINC08551088 & -09.90 \\
ZINC08551088 & -11.70 & ZINC85574911 & -09.80 \\
ZINC85534368 & -11.60 & ZINC44555643 & -09.80 \\
ZINC94303217 & -11.60 & ZINC43411699 & -09.80 \\
ZINC87515504 & -11.50 & ZINC13829348 & -09.80 \\
ZINC85564649 & -11.50 & ZINC13829346 & -09.80 \\
\hline
\end{tabular}

Figure S4. A) Experimental pose of acetyl-CoA (magenta: 1GHE) superimposed on the experimental pose (blue: 1QSM), showing differences in the conformation having optimal binding interactions. 1 GHE protein is represented as cyan colour cartoon. B) Redocked pose of acetyl-CoA (magenta) superimposed on the experimental pose (blue: 1QSM) of yeast Hpa2.

A)

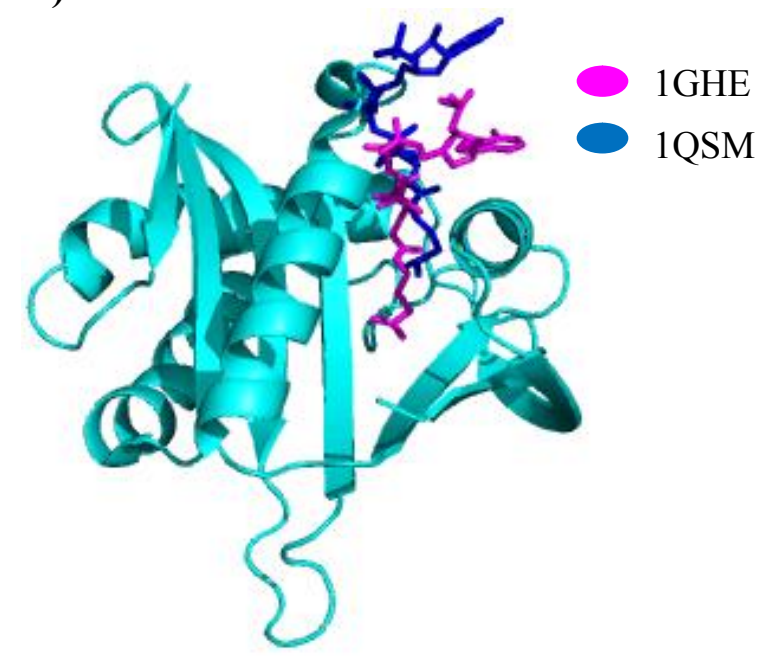

B)

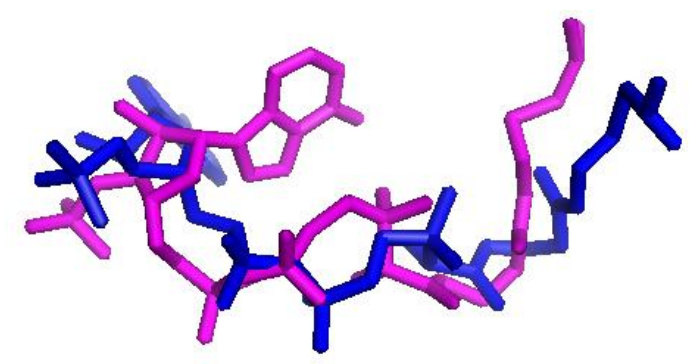

1QSM

Redocked 
Figure S5A. Structures of best ten compounds showing good binding with monomer in virtual screening.

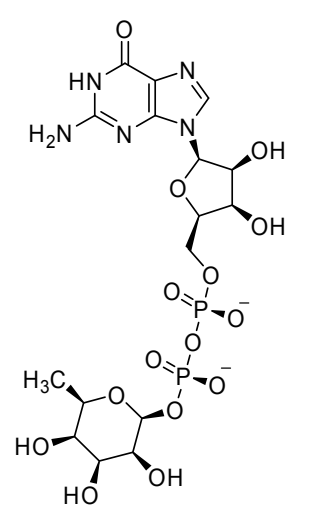

ZINC43411693

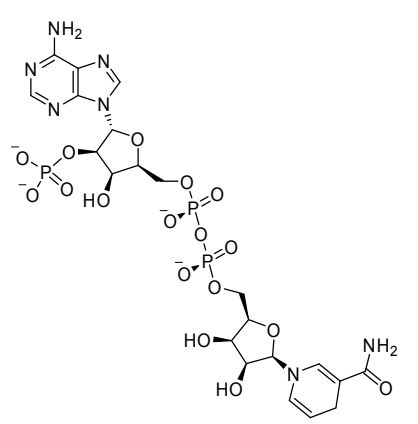

ZINC85574911

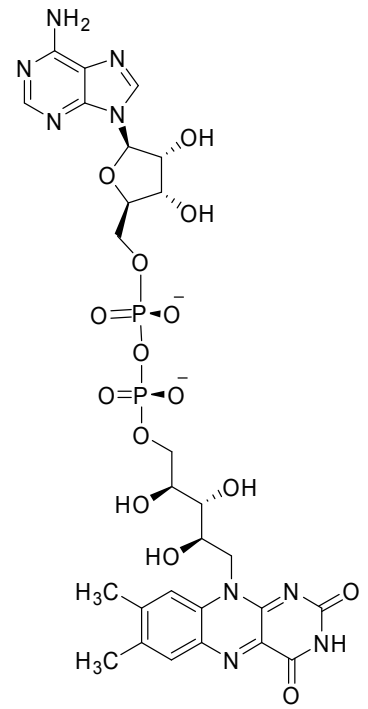

ZINC85629877

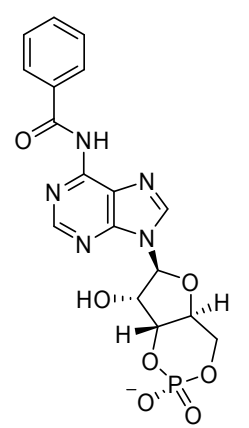

ZINC44559643

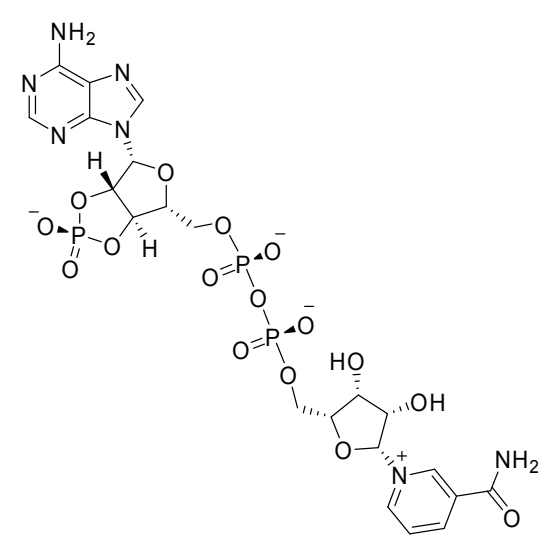

ZINC94303217

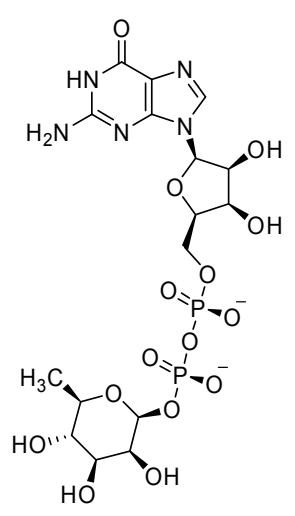

ZINC43411699

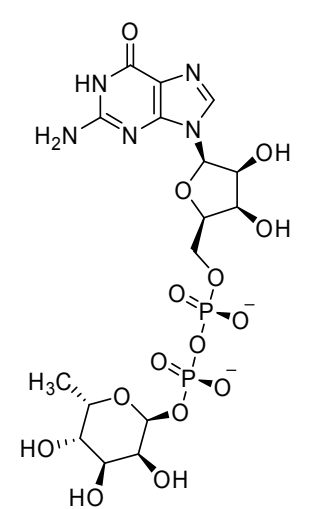

ZINC43411702

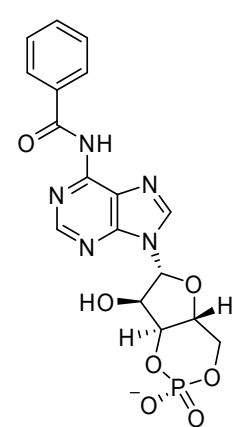

ZINC13829348

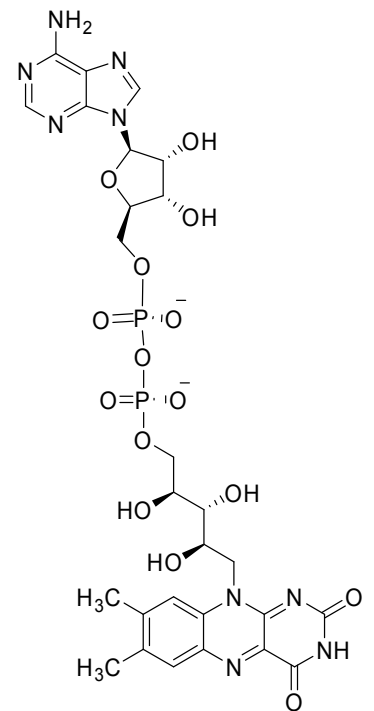

ZINC08551088

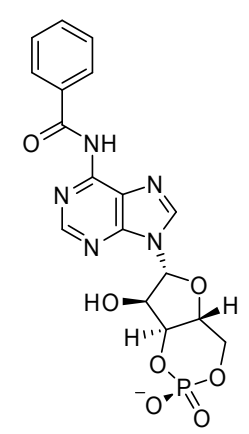

ZINC13829346 
Figure S5B. Structures of best ten compounds showing good binding with dimer in virtual screening.

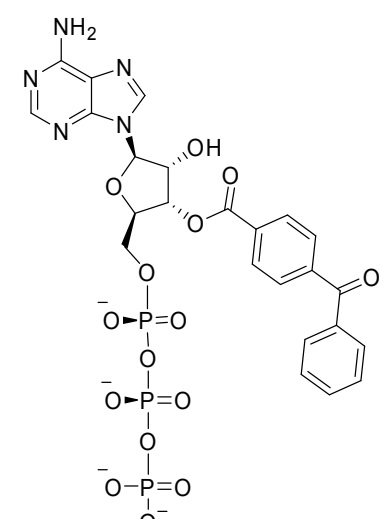

ZINC65731330

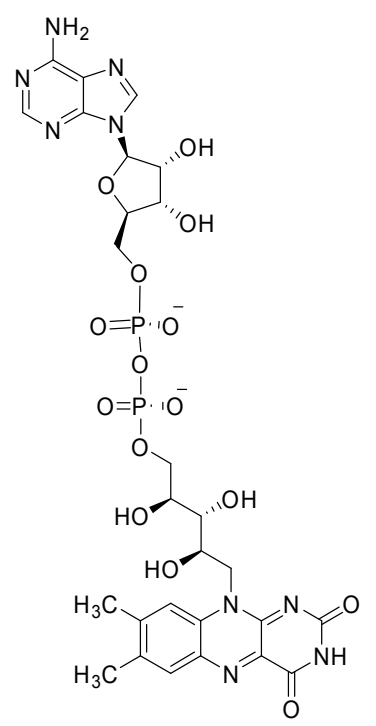

ZINC08551088

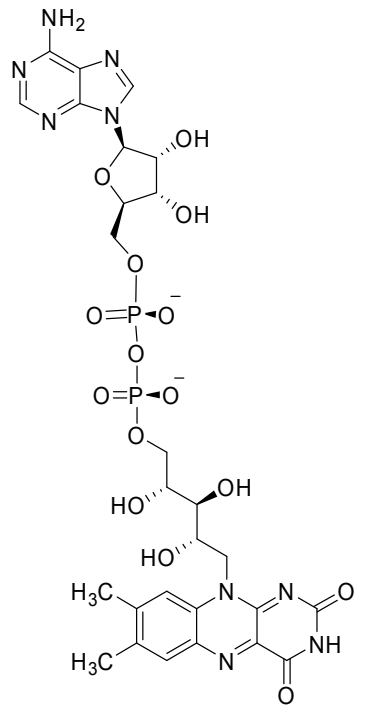

ZINC08215434

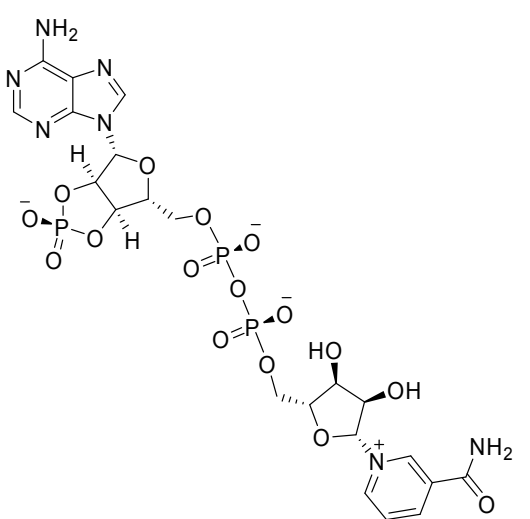

ZINC85534368

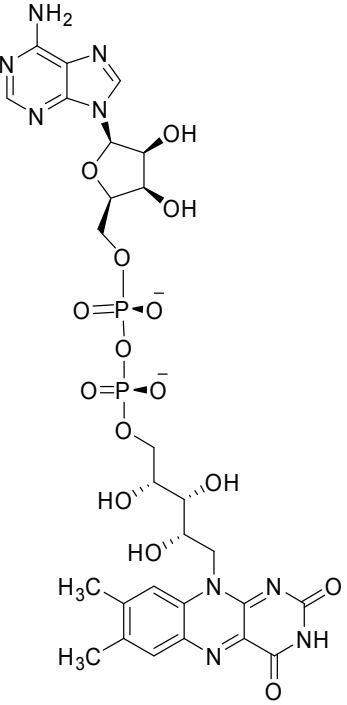

ZINC85564657

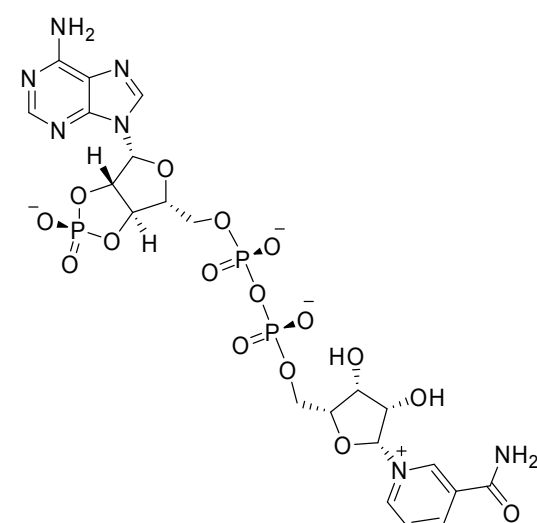

ZINC94303217

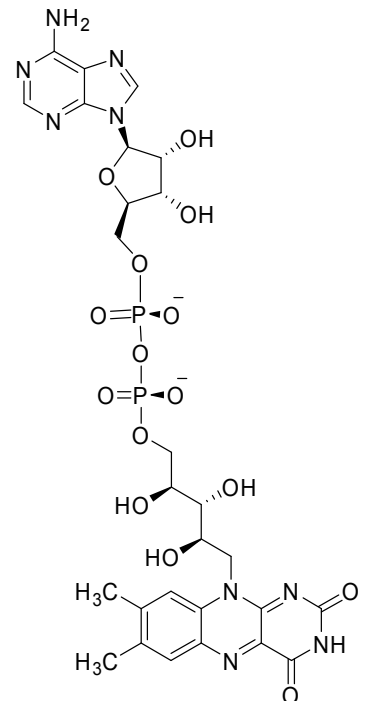

ZINC85629877

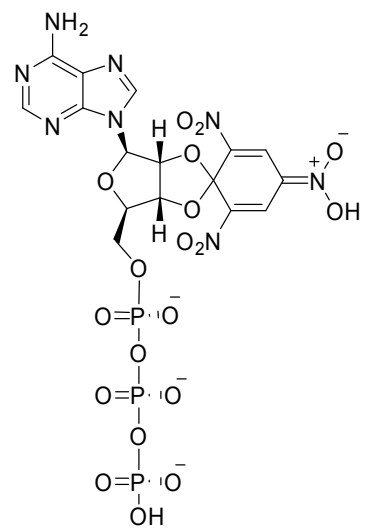

ZINC87515504

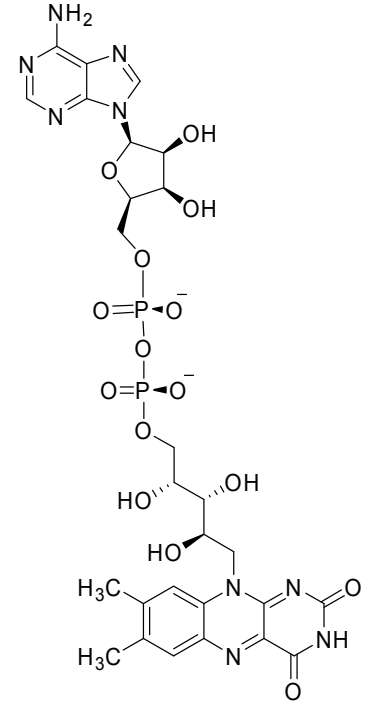

ZINC85564663

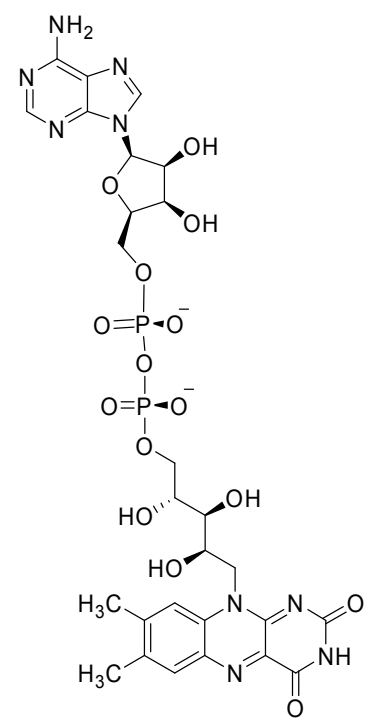

ZINC85564649 
Figure S6. Redocked pose of acetyl-CoA (magenta) superimposed on inhibitors docked pose (blue: ZINC08215434, green: ZINC85629887, cyan: ZINC65731330 and marine: ZINC85564657) at the model active site (green cartoon) to depict the similarity in binding interactions.

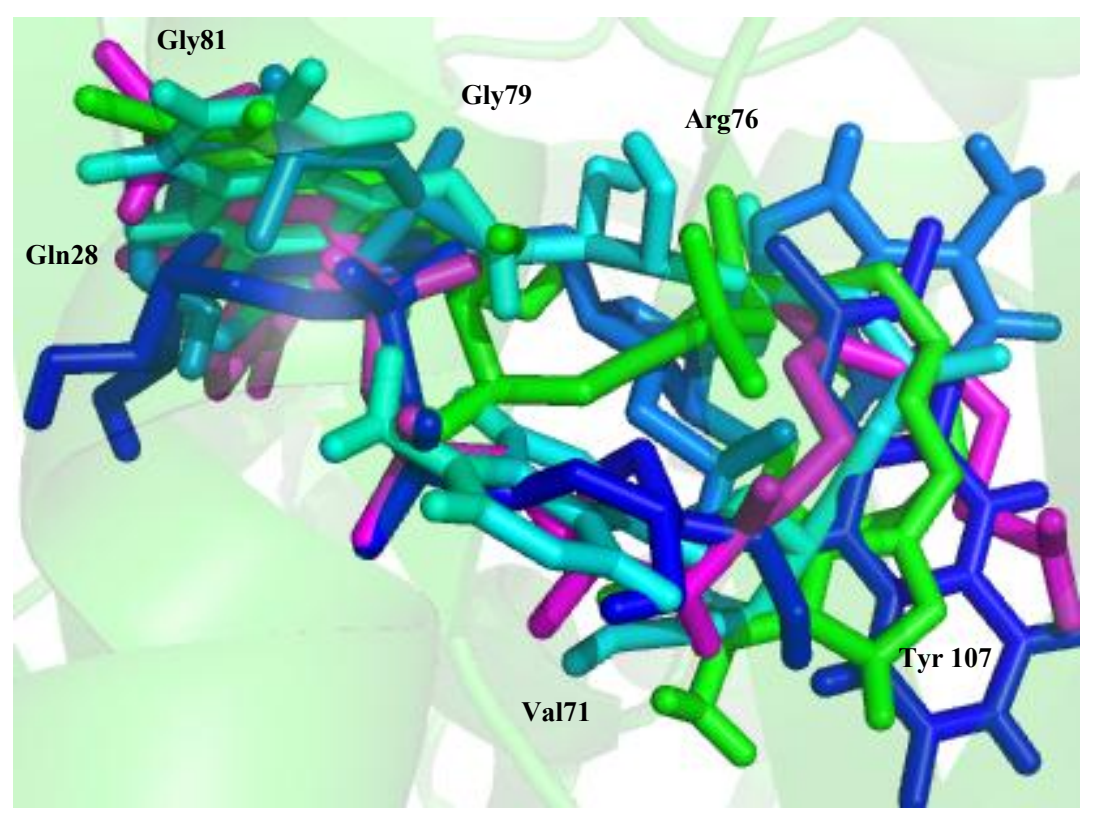

